\title{
Introduction to Chapter 7
}

Sarmiento Barletti's introduction of a tool for use in multi-stakeholder forums (MSFs) follows on naturally from Chapter 6, which looked at the evolution of MSFs within ACM efforts (in Bolivia, Nicaragua and Ghana). Sarmiento Barletti, whose ACM research began later than most contributors, initially reports the findings of a realist synthesis review of the MSF literature, thereby establishing some key parameters that affect the success or failure of an MSF.

Based on this review plus the team's own examples from their research in Brazil, Ethiopia, Indonesia and Peru, he concludes that rather than "designing engagement" in a way that overcomes a given context, we need to begin "designing for engagement" in a way that acknowledges and builds on the local context and treats local peoples as partners rather than mere "beneficiaries."

Sarmiento Barletti then turns to his team's experience developing and later testing a tool designed to address the power inequities that are recognized - but are then often ignored - in so many extant MSFs: How are we doing? Using three cases, two in Peru, one in Indonesia, the research team and MSF participants collaborated to explore how they could address the inequities and exclusions they identified within their MSF and local setting.

This chapter also raises the relevance of ACM and good MSF approaches to better management of extractive industries, a rarity in discussions of such collaborative actions and research. It also proposes MSFs as a field in which to continue developing and adapting ACM. 